\title{
OpenCapSense: A Rapid Prototyping Toolkit for Pervasive Interaction Using Capacitive Sensing
}

\author{
Tobias Grosse-Puppendahl*, Yannick Berghoefer ${ }^{\dagger}$, Andreas Braun*, Raphael Wimmer ${ }^{\ddagger}$, Arjan Kuijper* \\ * Fraunhofer Institute for Computer Graphics Research IGD, Darmstadt, Germany, \\ \{tobias.grosse-puppendahl, andreas.braun, arjan.kuijper\}@igd.fraunhofer.de \\ ${ }^{\dagger}$ Technical University Darmstadt, Darmstadt, Germany, yannick.berghoefer@stud.tu-darmstadt.de \\ $\ddagger$ University of Regensburg, Regensburg, Germany, raphael.wimmer@ur.de
}

\begin{abstract}
Capacitive sensing allows the creation of unobtrusive user interfaces that are based on measuring the proximity to objects or recognizing their dielectric properties. Combining the data of many sensors, applications such as in-the-air gesture recognition, location tracking or fluid-level sensing can be realized. We present OpenCapSense, a highly flexible opensource toolkit that enables researchers to implement new types of pervasive user interfaces with low effort. The toolkit offers a high temporal resolution with sensor update rates up to $1 \mathrm{kHz}$. The typical spatial resolution varies between one millimeter at close object proximity and around one centimeter at distances of $35 \mathrm{~cm}$ or above.
\end{abstract}

Keywords-capacitive sensing; pervasive interfaces; proximity sensing; smart environments;

\section{INTRODUCTION}

In recent years, pervasive interaction technologies like body parameter sensing and gesture recognition replaced input modalities such as keyboard and mouse in many applications [7]. The realization of such new user interfaces requires one to consider a variety of interaction modalities. Besides different multi-touch and camera-based interaction systems, the research area of capacitive sensing for pervasive interaction gains increasing interest.

Employing this technology, it is possible to implement interfaces that are able to determine gestures, body movements and environmental changes at typical distances up to $50 \mathrm{~cm}$ [16]. In contrast to camera-based methods, capacitive sensing has the advantage of being robust against changing lighting conditions and visual occlusion. Additionally, capacitive sensors have significantly lower impact on a user's perceived and actual privacy, compared to optical tracking methods. Energy-efficient sensors can be deployed unobtrusively underneath furniture, carpets or within walls. Sensed data can be processed with computationally cheap algorithms. However, the drawbacks are a limited resolution and error-proneness in environments with many conductive objects or electrical devices that affect electric fields. Using capacitive proximity sensors, reasearchers have realized location tracking systems [18], [17], wearable activity recognition systems [3], [10], [4], smart furniture [11], [20] and

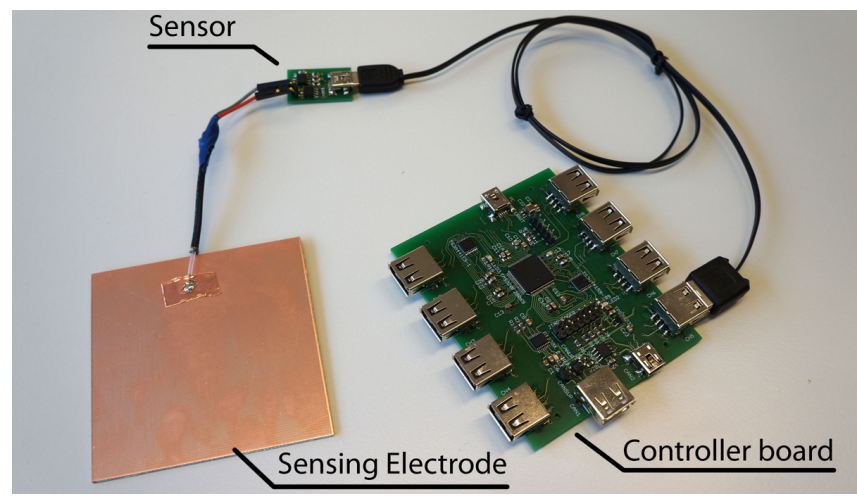

Figure 1. The OpenCapSense board supports eight capacitive touch and proximity sensors. Moreover, it integrates various communication interfaces such as Controller-Area-Network for creating sensing arrays.

gesture recognition systems [19], [16].

Currently, it is challenging for researchers to experiment with capacitive proximity sensing as there are few publically available hardware solutions. Moreover, data on measurement resolution is rarely available, distances and measurement techniques. OpenCapSense, shown in Figure 1 , is a novel open-source toolkit for capacitive sensing and is presented in this paper. It allows capturing and analyzing data from a multitude of capacitive sensors at update rates of up to $1 \mathrm{kHz}$. This enables prototyping novel pervasive real-time applications based on capacitive sensing, such as multi-object tracking and fall detection.

In this paper we present the following contributions:

- We introduce a novel open-source ${ }^{1}$ toolkit for capacitive sensing, providing eight sensor channels and two combinable sensor types.

- The system is evaluated with various sensing electrode sizes as well as opaque and transparent materials.

- We demonstrate practical uses of OpenCapSense in different applications scenarios, including a gesture recognition device and a fall detection system.

\footnotetext{
${ }^{1}$ Hardware licensed under Creative Commons CC BY-NC-SA 3.0, software licensed under GPL v3
} 


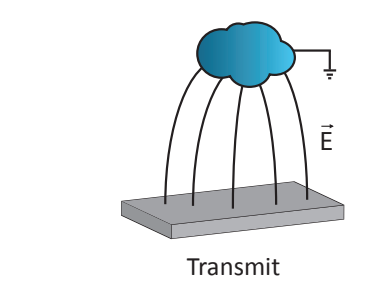

Loading Mode

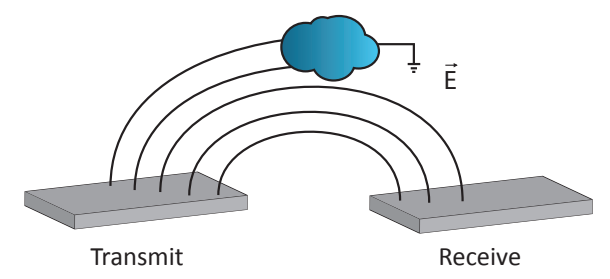

Shunt Mode

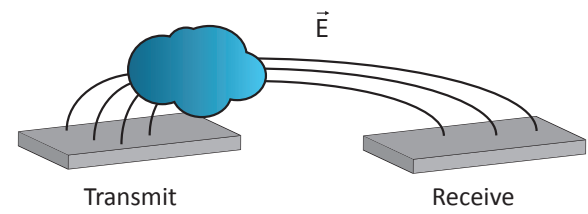

Transmit Mode

Figure 2. Measurement modes identified by Smith [16]

\section{BACKGROUND \& RELATED WORK}

Capacitive sensing is an established and well understood sensing technology. Considering pervasive user interfaces, it is possible to recognize the presence of human body parts, such as fingers, hands, even allowing whole-body interaction. A recent project investigates Swept Frequency Capacitive Sensing to realize touch interaction on various materials and surfaces [13]. In contrast to some electronics toolkits that support capacitive touch sensing [9], [14], capacitive proximity sensing requires a significantly better resolution. The first practical application of capacitive proximity sensing dates back almost a century, to the Russian physicist Leon Theremin, who invented the first electronic music instrument in 1919. Recent scientific contributions in this area include the work by Smith et al., who applied the measurement technique to realize gesture recognizing interfaces for human-computer-interaction [16], [15]. A few years later, the CapToolKit [19] was released, an easy-to-use prototyping platform for proximity sensing applications that has been used by several research groups to realize applications, such as activity recognition and full-body interaction [19]. The kit consists of a circuit board that incorporates a microcontroller and interfaces to connect up to eight sensors that are attached using regular USB connectors. Although several tutorials on capacitive proximity sensing can be found online ${ }^{2}$, these generally require soldering and programming. Moreover, data on the achievable detection range as well as spatio-temporal resolution is rarely published.

Smith [16] distinguishes three capacitive measurement modes that are outlined in Figure 2. Loading mode relies on measuring the displacement current caused by the presence of a grounded object in proximity of a single transmitting electrode. The main advantage of this mode is that electrodes can be placed arbitrarily and can be easily shielded against the influence of existing electric potentials in the neighborhood. Exemplary applications that rely on this sensor mode are capacitive tables, shelves and couches [20], [11]. The second method, the shunt mode, is based on the principle that a grounded body part affects the electric field between a transmitter and a receiver electrode. In this

\footnotetext{
${ }^{2}$ e.g. http://arduino.cc/playground/Main/CapacitiveSensor
}

mode, each sensor electrode can be configured as either transmitter or receiver. Measurements can be conducted between all receiver-transmitter combinations, resulting in $\frac{n \cdot(n-1)}{2}$ distinct measurements for $n$ electrodes [16]. This is particularly suited for applications that require a high number of measurements to gain precision, e.g. gesture recognition of one or more hands [16]. The third method is called transmit mode and can determine proximity of a human body by coupling a person to a changing electric potential and receiving the signal using a grounded sensor electrode as the receiver. Transmit mode requires a user to be connected to an electrode which is only applicable in certain scenarios, like user identification at multi-touch tables [6].

\section{OpenCapSense Rapid Prototyping Toolkit}

We have developed our own capacitive proximity sensing toolkit - OpenCapSense - that enables researchers to rapidly prototype capacitive proximity sensing applications, such as pervasive user interfaces for whole-body interaction or activity recognition. Similar requirements have driven the development of the CapToolKit, resulting in analogous design decisions [19]:

- Open-source hardware and software allows others to improve and customize our design

- Plug-and-play operation with a simple text-based protocol for configuring the device and reporting measurement data

- Flexible and customizable electrode layouts

- Reduction of stray capacitance and external noise by putting the sensor circuit as close to the electrode as possible

- Use of affordable and easily obtainable hardware components

We have identified three major limitations of the CapToolKit that have driven the design of the OpenCapSense toolkit:

- CapToolKit only supports loading mode sensing. OpenCapSense additionally supports transmit and shunt mode sensing. These modes decouple sending and receiving electrode and allow realizing applications with a higher detection range and a better temporal resolution due to parallel transmitter operation. 
- The platform has a low update rate of approximately $50 \mathrm{~Hz}$ due to its slow 8-bit microcontroller and software architecture. The update rate is further reduced when multiple sensors are connected. OpenCapSense offers an update rate between $1 \mathrm{kHz}$ and $250 \mathrm{~Hz}$ when using all sensors. This is sufficient for tracking very fast motion, for example in fall detection scenarios.

- CapToolKit is not suitable for creating larger sensing arrays, because it requires to individually connect each controller board to a host PC. As CapToolKit boards are not synchronized with each other, active sensors may negatively affect adjacent other sensors. We address this issue by providing two real-time bus interfaces that can be used for synchronization and data exchange.

OpenCapSense consists of a board employing a microcontroller that is tailored to complex real-time calculation and control tasks. The sensors can be combined freely with little configuration effort, enabling application developers to use different measurement modes or combine them to realize hybrid measurements. Moreover, the software provided with OpenCapSense provides functionality from evaluating sensor measurements up to realizing a specific user interface using machine-learning techniques.

\section{A. OpenCapSense Board}

The controller board is built around a TI TMS320F28069 32-bit microcontroller with native floating point unit and provides eight sensor channels for measurements. Sensors are connected to the board by a standard USB cable. A single sensor channel represents a generic interface that supports different sensor types. Apart from two power supply lines, there are two software-configurable lines for communicating with a sensor. The first line leads to a microcontroller's general-purpose-input-output port (GPIO) while the second line can be switched to either an analog-to-digital converter (ADC) or a time-capturing unit. This architecture enables us to dynamically configure the toolkit's sensor channels for different sensor types. For example, digital output signals, like frequency-modulated rectangular pulses, benefit very much from time-capturing units that can measure time intervals between the rising and falling edges of a signal. The time-capturing units analyze signals with a temporal resolution of $12.5 \mathrm{~ns}$. Analog receivers are usually connected to an ADC that samples the signal at high data rates up to $100 \mathrm{kHz}$ and 12 bit resolution. This method allows for obtaining a set of values representing the signal's amplitude in a certain timespan.

Pervasive interfaces are often driven by a variety of different sensors, such as accelerometers or temperature sensors. These sensors can be interfaced using an expansion header driven by an Inter-Integrated Circuit $\left(\mathrm{I}^{2} \mathrm{C}\right)$ bus. The board also includes a Controller Area Network (CAN) interface for realizing larger sensing arrays and simplify prototyping in automotive applications, where CAN is ubiquitously used.
The board can be connected to a computer using USB, providing both power and a communication interface for configuration and data exchange.

\section{B. OpenCapSense Sensors}

We have created two exemplary sensors that can be applied to realize all measurement types. Loading mode sensors are especially suitable for implementing larger sensing systems for activity recognition or whole-body interaction [20]. Shunt mode sensors can be applied to realize gesture recognition interfaces with a high spatial resolution [11]. Using transmit-mode measurements, we can distinguish between different users. This measurement mode can be realized applying our shunt mode sensors.

1) Loading Mode Sensor: The loading mode sensor is based on a timer configuration called astable multivibration [8]. The timer controls the charging and discharging cycles of the capacitor that is created by the sensing electrode and the surrounding environment, e.g. a person's limb or body. It toggles succeeding charging and discharging cycles at the time when an upper or lower threshold voltage at the virtual capacitor is reached. In order to measure the capacitance in a certain direction and prevent disturbances from objects nearby, a shield electrode can be placed directly underneath the measuring electrode. The shield is driven with the same potential as the sensing electrode, such that the capacitance between the two electrodes is negligible.

2) Shunt Mode Sensor: In shunt mode, a receiver electrode is used to measure the displacement current from a transmitter electrode [16]. When a human body part enters the electric field, the field between a transmitter and receiver is interrupted. This results in a decrease of displacement current and thus a decreasing capacitance between the transmitter and receiver. Due to separate transmitter and receiver electrodes, shunt mode offers the possibility to perform parallel measurements using multiplexing approaches [16]. The shunt mode sensor measures the displacement current floating from a transmitter electrode to the receiver electrode.

\section{OpenCapSense Software}

We have developed a generic software framework called SenseKit that has an interface to OpenCapSense [11]. It analyzes the inputs of the virtual serial port created by the OpenCapSense board. SenseKit has a generic processing pipeline for classification and evaluation, it supports windowing, feature extraction and different classifiers. The strong integration of the WEKA framework ${ }^{3}$ eases the development of applications relying on machine-learning, such as activity recognition. Therefore, classes can be recorded as training data and then evaluated in real-time classifications. Moreover, platform-independent OpenCapSense APIs for C and Java can be used for developing own applications.

\footnotetext{
${ }^{3}$ http://www.cs.waikato.ac.nz/ml/weka/
} 


\section{Evaluation}

In order to analyze the capabilities of the OpenCapSense toolkit, we have conducted different experiments investigating the behavior of our loading and shunt mode sensors. There are two essential factors that can characterize this behavior. The first factor is the maximum spatial distance between a sensor electrode and a detectable object at which a presence might be registered. The second factor is the signal-to-noise ratio that can be affected by electromagnetic interference, thermal noise or ambient temperature variation. We combine both factors to derive the spatial resolution of our system.

Considering the broad spectrum of applications for capacitive sensors, we also investigated different electrode materials. Copper, which combines excellent surface resistance and low cost is the material most commonly used for electrode design. However, in many applications, such as gesture recognition, it is desirable to realize capacitive proximity sensing on transparent surfaces like windows or displays.

Various materials can be applied in those scenarios. For example, a foil of polyethylene terephthalate (PET) coated with indium tin oxide (ITO) provides both high conductivity and high transmittance of light at visible wavelengths. Another interesting material is PEDOT:PSS, an electrically conductive material made from polymers. It is used in different research disciplines like printed field-effect transistors and photovoltaic cells. Since it can be applied to different materials using ink-jet printing, it promises very good properties for rapidly prototyped sensing electrodes [5], [1]. Both variants can be used to create printable transparent conduction films that offer manifold alternatives for sophisticated sensor designs and rapid-prototyping.

\section{A. Test setup}

As mentioned in the introduction, the capacitive proximity sensing toolkit presented by Wimmer et al. [19] is a commonly used platform for implementing capacitive loading mode applications. Only few capacitive sensing systems were evaluated in a quantitative manner by presenting data related to the sensor detection range and spatial resolution. In their work about CapToolKit, Wimmer et al. adopted a measuring arrangement presented by Zimmerman et al. [21]. Considering this well-defined test setup, we evaluated OpenCapSense's and CapToolKit's capabilities under comparable prerequisites and circumstances that are described in the following.

Regarding the different properties of human arms and hands, e.g. shape and conductivity, it is obvious to use a standardized measuring body. Such an object should have properties similar to a human arm and be easily applicable to different experiments. Zimmerman et al. [21] introduced a grounded aluminum tube with a length of approximately 48 $\mathrm{cm}$ and a diameter of $8 \mathrm{~cm}$ that acts as a surrogate arm. They

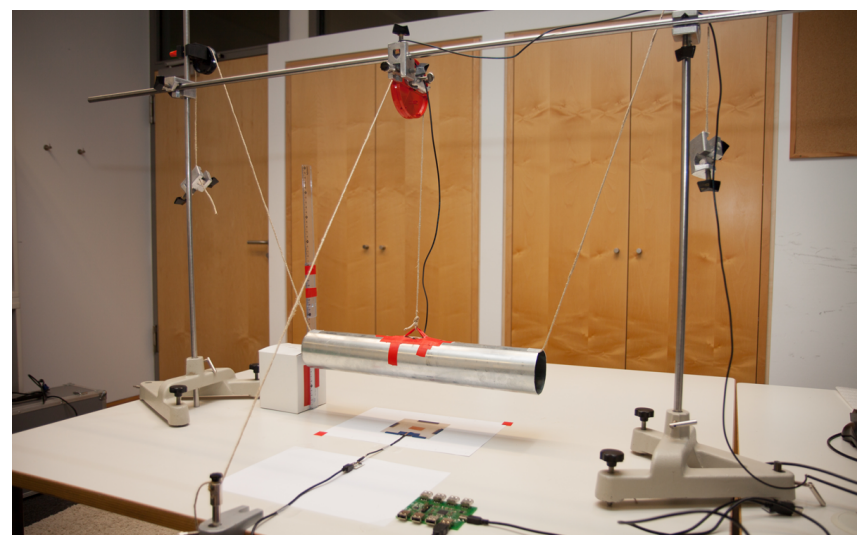

Figure 3. Test setup to determine loading mode sensor's resolution and behavior related to different electrode materials and sizes. The tube acts as a surrogate arm and can be lifted up and down.

showed that this tube can be an appropriate replacement for a human arm, delivering slightly deviant measurement results. Based on this description, we developed a test setup that is shown in Figure 3.

\section{B. Spatial resolution}

As the descriptions of previous measurements [19], [16] provide only little information on how the resolution of a capacitive proximity sensor can be measured, we introduce a principle to determine a system's spatial resolution. The normalized sensor values related to the distance of the aluminum tube are determined with a series of measurements. We adjust the aluminum tube to distances from $0 \mathrm{~cm}$ up to $40 \mathrm{~cm}$. For every distance $d$, a series of samples is recorded for 10 seconds. This allows calculating basic statistical values of the sampled sensor measurements. The most important values are the arithmetic mean $\overline{s_{n}}(d)$ and the standard deviation $\sigma_{n}(d)$, the latter being the main criterion to determine the system's signal-to-noise ratio.

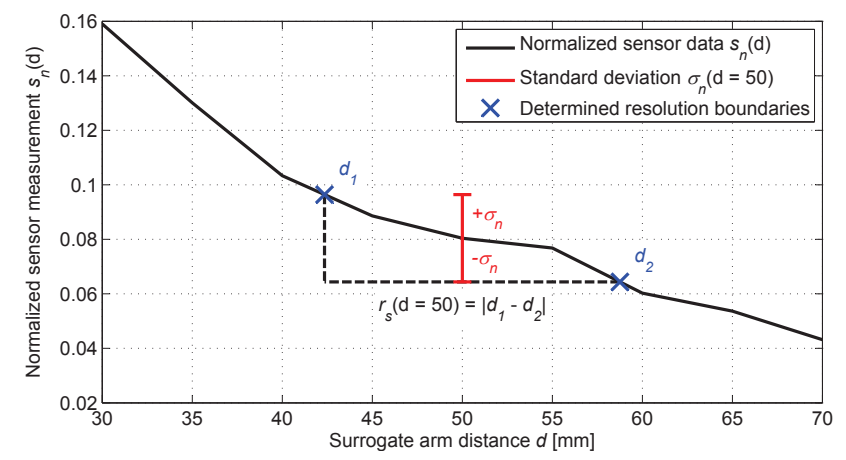

Figure 4. The approach to determine the spatial resolution of a sensor configuration is based on a linearly interpolated measurement series and the standard devition $\sigma_{n}(d)$ at a given point. 


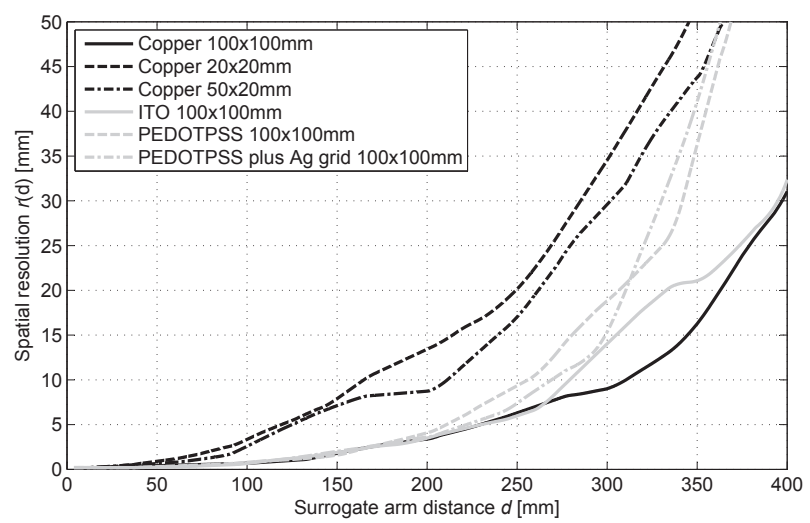

Figure 5. Spatial sensor resolution of a loading mode sensor in relation to the surrogate arm distance.

Figure 4 outlines the way of obtaining the spatial resolution $r_{s}(d)$ for any given distance $d$. As a first step, the measurement series $s_{n}(d)$ and $\sigma_{n}(d)$ are linearly interpolated. For a distinct distance $d$ the standard deviation $\sigma_{n}(d)$ is used to look for distances $d_{1}$ and $d_{2}$ that provide measurement values that deviate from $s_{n}(d)$ within the environment of $\pm \sigma_{n}(d)$. The result is an estimation of the spatial resolution $r_{s}(d)$, which is finally calculated as the absolute difference between these distances. Effectively, this means the spatial resolution is good if the resulting value is small. The spatial resolution is an indicator for the precision $\pm r_{s}(d)$ a capacitive sensor is able to detect a defined object at a certain distance $d$.

\section{Performance evaluation and influence of electrode mate- rials}

Using $r_{s}$ as the main characteristic value of a capacitive sensor system, it is reasonable to investigate if different electrode surface materials result in diverging spatial resolutions. Additionally we investigate the dependency between the size of a sensing electrode surface and the resulting spatial resolution, which provides essential information that is required when prototyping capacitive sensor applications. The sensing electrodes test set contains rectangular shaped copper electrodes of various sizes. Furthermore, we compare copper electrodes to electrodes made of ITO and PEDOT:PSS. Using the introduced test setup and measurement method, the normalized sensor characteristics and the spatial resolution were determined.

As the spatial resolution is calculated on interpolated curves of the sensor characteristics and their corresponding standard deviation, its raw curve is not uniform. Therefore the $r_{s}$ curves were smoothed, which allows for comparable inferences on the resolution.

Regarding the loading mode sensor, we evaluated the influence of different electrode materials and sizes. The final results for a measurement window of $10 \mathrm{~ms}$ are shown in

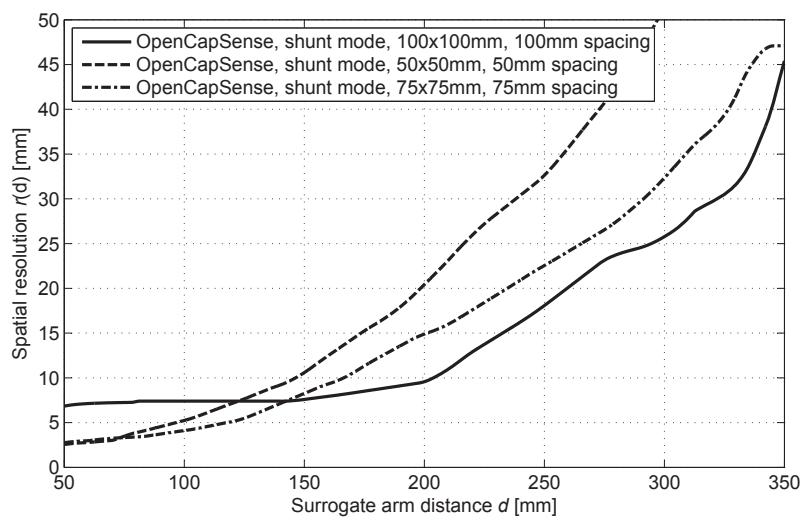

Figure 6. Spatial sensor resolution of a shunt mode sensor in relation to the surrogate arm distance.

Figure 5. As expected, larger electrode sizes provide a better resolution than smaller ones. At distances of $20 \mathrm{~cm}$, the resolution of a $100 \times 100 \mathrm{~mm}$ copper electrode is almost four times higher than the resolution of an electrode with a size of $20 \times 20 \mathrm{~mm}$. Regarding different materials, transparent ITO electrodes perform almost as well as copper electrodes. Although ITO has a higher surface resistance than copper, the influence of that property turns out to be of low significance due to the very small displacement current flowing from the electrode to grounded parts in the environment. PEDOT:PSS showed similarly good resolution for near distances. At distances above $30 \mathrm{~cm}$ the resolution decreases strongly, which can be attributed to the non-uniform application of the polymeric conductor on the PET caused by inkjet printing. We can conclude that larger electrodes perform significantly better in loading mode when detecting objects at great distances. The transparent properties, easy inkjet printing and the good performance of PEDOT:PSS qualifies the conductor for rapidly building prototypical user interfaces.

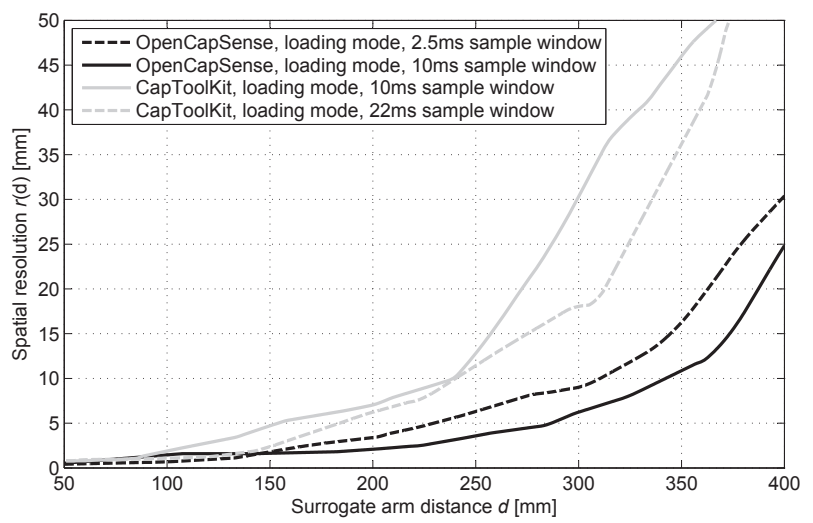

Figure 7. Comparison between spatial resolutions of OpenCapSense and CapToolKit [19] using a $100 \times 100 \mathrm{~mm}$ copper electrode. 
In later development stages, ITO can provide a very good performance comparable to copper.

The evaluation results for a shunt mode sensor with a measurement window of $20 \mathrm{~ms}$ are illustrated in Figure 6. The results show that smaller electrode sizes provide a better performance at low distances, while bigger electrode sizes are more suitable for detecting objects at high distances. It is notable that the measurement object turns into transmit mode at object distances below $5 \mathrm{~cm}$, letting the sensor values increase again. This distance depends on the electrode size: when using small electrodes with a size of $50 \times 50 \mathrm{~mm}$, transmit mode comes into effect at approximately $2 \mathrm{~cm}$, while the distance increases to about $5 \mathrm{~cm}$ on larger electrodes sized $100 \times 100 \mathrm{~mm}$.

\section{Comparison to existing solutions}

Based on our experiment setup, we evaluated the loading mode sensor for both, CapToolKit and OpenCapSense, using the same methodology. Figure 7 shows a comparison of different sampling windows. As expected, the resolution of both, OpenCapsense and CapToolkit, increases for longer sampling windows. Comparing both $10 \mathrm{~ms}$ measurement series, OpenCapsense shows a distinct improvement to CapToolkit. For distances of $200 \mathrm{~mm}$, OpenCapSense improves the resolution by the factor three compared to CapToolKit. Regarding a reduced sample window of $2.5 \mathrm{~ms}$ the resolution is robust and higher than CapToolKit. As CapToolKit supports minimum measurement windows of $10 \mathrm{~ms}$, a smaller window could not be selected for comparison with OpenCapSense. It can be concluded that OpenCapSense's temporal and spatial resolution is a significant improvement to CapToolKit and allows investigating new pervasive application scenarios with very fast update rates.

\section{Application EXAmples}

In order to demonstrate the capabilities of the OpenCapSense toolkit and to observe its properties in real application scenarios, we built a variety of prototypical systems that we will present in the following.

\section{A. Smart couch}

We have realized a smart couch (shown in Figure 8) that can sense the postures of up to two users [11]. Therefore, we applied eight loading mode sensors that were hidden under the upholstery of the smart couch. Two electrodes were placed under both arm rests, two at the backrest and four electrodes under the seating cushion. The electrodes under the armrests and in the backrests measure the distance of a person's body through a thick wooden plate and the upholstery. The electrode sizes were chosen to be as big as possible, resulting in electrodes with the size of $30 \times 10 \mathrm{~cm}$ underneath the armrests and electrodes with a size of $30 \times 20 \mathrm{~cm}$ under the seating cushions and backrests.
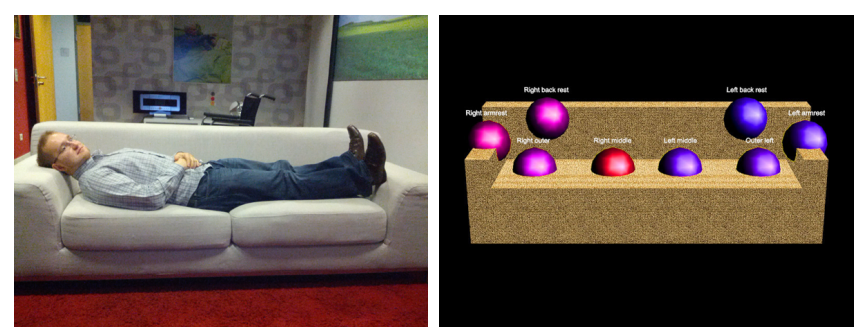

Figure 8. The smart couch can recognize 10 different postures with a very high accuracy of $97.5 \%$. The right image shows the sensor distribution and the corresponding values, blue spheres denote less influence from objects than red spheres.

The OpenCapSense board performs a series of measurements and then passes it to our software framework SenseKit for feature extraction and classification. Using WEKA's RBF network classifier, we can obtain a classification accuracy of more than $97.5 \%$ for 10 different postures. This test was performed using the recordings of 18 different subjects, splitting the training and test data sets into 9 subjects each.

\section{B. Gesture recognition}

Gesture recognition is one of the mostly applied application scenarios that has been evaluated for capacitive proximity sensors [19], [21], [2]. The unobtrusive applicability allows detecting gestures over a distance through nonconductive materials. The position of one or more hands can be determined using different analytical or probabilistic models.

A prevalent issue, present in all publications above, is the speed in which the hand locations are determined - a factor that greatly influences the perceived quality of interaction. Delays that surpass real-time are considered unacceptable [12]. Regarding OpenCapSense's sophisticated processing capabilities, gesture recognition applications can be implemented on the demonstration board itself. Combined with a high sampling rate this enables real-time hand gesture

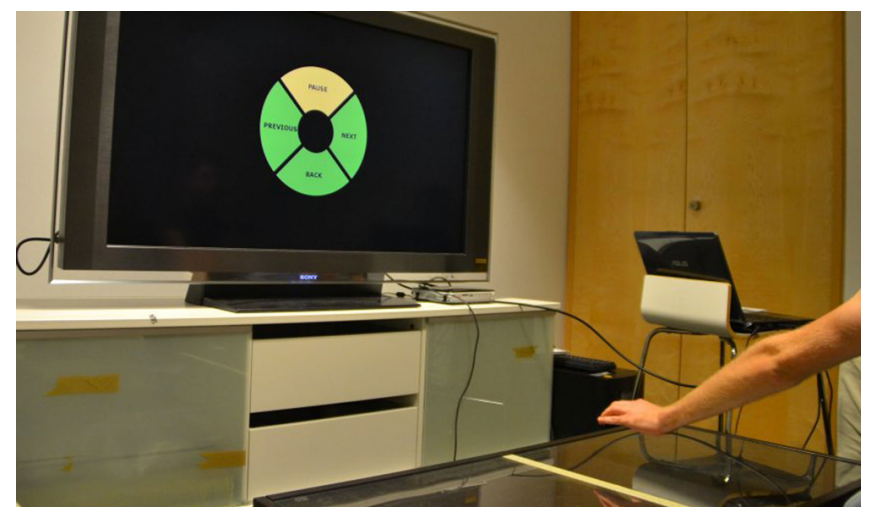

Figure 9. The CapTap controlling a multimedia application with radial menus. It is based on an array of OpenCapSense boards and an accelerometer to detect knocking and tapping. 

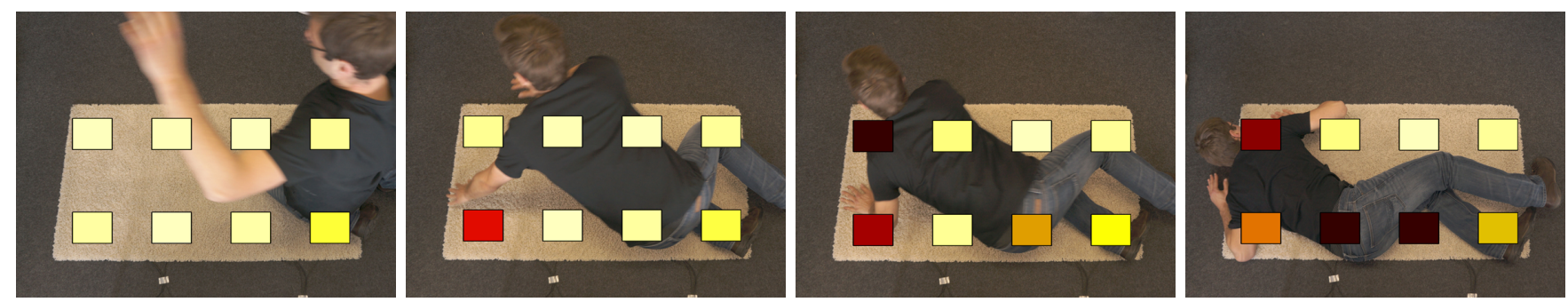

Figure 11. Fall detection can greatly benefit from very high sensor update rate (1.2 ms measurement window per sensor): red sensor values denote near measured distances and white sensor values represent no body parts in the proximity of the sensor. The simulated fall situation lasted approximately $800 \mathrm{~ms}$.

recognition while preserving a good precision. A recent prototype we are developing is the CapTap - a combined hand tracking and knock detection system that uses multiple OpenCapSense boards configured as a sensing array and a single accelerometer interfaced through $\mathrm{I}^{2} \mathrm{C}$. The system, shown in Figure 9, allows controlling typical multimedia applications with selection indicated by hand position and actions triggered by different knocking events that are registered by the accelerometer.

\section{Wearable devices}

In the area of activity recognition, capacitive sensors were applied to measure muscle contractions that can lead to a more accurate classification of the activity performed [3]. Grosse-Puppendahl et al. have extended a wrist-worn activity logger (shown in Figure 10) with an OpenCapSense loading mode sensor [10]. The sensor's electrode with the size of $10 \times 2.6 \mathrm{~cm}$ is integrated unobtrusively in the wristband and is able to measure the distance to objects nearby. This information can help identifying activities like drinking, when the hand approaches and moves away from a table. Moreover, knowledge about the type of object can be retrieved, for example by recognizing that a hand is covered by a blanket when sleeping. The prototypical system was evaluated with 9 activities of daily living, such as preparing

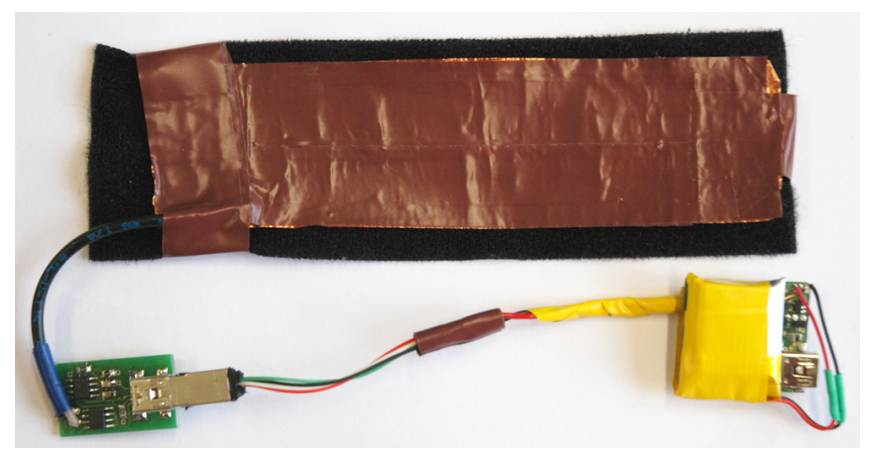

Figure 10. A wrist-worn accelerometer employing a proximity sensor with an invisible electrode in the wristband can enhance activity recognition by providing information about the object characteristics and their proximity [10]. bread, sleeping and eating. Integrating the knowledge of capacitive proximity sensors and applying a Support Vector Machine with a very easy feature set could enhance the classification accuracy by $6.3 \%$ to $73.5 \%$ [10].

\section{Fall detection}

Many pervasive environments require affordable and accurate methods for tracking humans. Capacitive proximity sensors are especially suited for this use case as they can be integrated unobtrusively into the environment. In real usecases, this monitoring requires to strongly consider privacy aspects. Researchers have therefore realized floor tiles and carpets employing capacitive proximity sensing that can be deployed in assistive environments and home automation scenarios [17], [18]. Smart floors can support the elderly and disabled persons in their activities of daily living, for example by monitoring emergency situations such as falls.

Current capacitive proximity sensing systems for localization and fall detection face the problem of having a very low update rate of $10 \mathrm{~Hz}$ or less [18]. Thus, a fall is usually detected by evaluating the time a person lies on the floor. However, a fall detection could benefit greatly from a very high update rate that enables a system to reconstruct the course of the fall precisely. We placed eight sensors and their corresponding electrodes $(30 \times 20 \mathrm{~cm})$ under an ordinary carpet. The smart carpet can detect a fall with a very high update rate of approximately $104 \mathrm{~Hz}$ for each sensor using a round-robin sensor scheduling. Therefore, we have reduced the measurement interval to $1.2 \mathrm{~ms}$. This very small measurement interval is associated with increased noise but can reliably detect a fall situation as shown in Figure 11.

\section{Vi. CONCLUSiOn \& Future Work}

We presented OpenCapSense: a highly flexible opensource toolkit that enables researchers to rapidly prototype pervasive applications based on capacitive sensors. Compared to previous toolkits OpenCapSense supports different measurement modes, provides a basic set of reliable signal processing algorithms, and can be easily interfaced via USB, $\mathrm{CAN}$, or $\mathrm{I}^{2} \mathrm{C}$. We provide libraries for Java and $\mathrm{C}$ that allow easy integration of OpenCapSense into existing projects and 
a strong integration of the WEKA framework to support machine-learning based post-processing of the sensor data.

The toolkit was evaluated for spatial resolution, the influence of different electrode materials on this metric, and comparatively analyzed with the CapToolKit - a platform that is widely used in research projects. Finally, we showed the broad applicability of the OpenCapSense Toolkit and capacitive sensors in general by presenting different studies, ranging from light wearable devices to fall detection systems in fixed installations. Still, these applications present only a small subset of potential scenarios that can be realized. We kindly invite interested researchers to use our system and further expand the horizon for capacitive sensing devices. The documentation, schematics, source code and evaluation results can be found at http://www.opencapsense.org.

In future work, we plan to support hybrid measurements that combine loading mode and shunt mode, which will improve the performance for various applications. Additionally, we want to simplify the integration of wireless systems by providing expansion cards based on either ZigBee or Bluetooth. In terms of applications a major interest is augmenting and improving our gesture recognition applications by adding more sensors and studying the associated effects. Finally, we will optimize the presented sensors, for example by designing more sophisticated transmitter and receiver frontends for shunt-mode sensors.

\section{ACKNOWLEDGEMENTS}

We would like to thank Sebastian Herber and Julian von Wilmsdorff for developing siginificant parts of OpenCapSense's firmware in a student project at Technical University Darmstadt. Moreover, we thank Rainer Berghoefer for the design and implementation of the evaluation setup. Special thanks go to Constanze Ranfeld, Institute of Printing Science and Technology at Technical University Darmstadt, for providing different sensing electrode material samples.

\section{REFERENCES}

[1] L. Basiricò, P. Cosseddu, a. Scidà, B. Fraboni, G. Malliaras, and a. Bonfiglio, "Electrical characteristics of ink-jet printed, all-polymer electrochemical transistors," Organic Electronics, vol. 13, no. 2, pp. 244-248, 2012.

[2] A. Braun and P. Hamisu, "Using the human body field as a medium for natural interaction," in PETRA '09, 2009, pp. 50:1-50:7.

[3] J. Cheng, O. Amft, and P. Lukowicz, "Active capacitive sensing: Exploring a new wearable sensing modality for activity recognition," in Pervasive '10, 2010, pp. 319-336.

[4] G. Cohn, D. Morris, S. Patel, and D. Tan, "Humantenna: using the body as an antenna for real-time whole-body interaction," in $C H I ' 12,2012$, pp. 1901-1910.
[5] X. Crispin, F. L. E. Jakobsson, A. Crispin, P. C. M. Grim, P. Andersson, A. Volodin, C. V. Haesendonck, M. V. D. Auweraer, W. R. Salaneck, and M. Berggren, "The origin of the high conductivity of (pedot-pss) plastic electrodes," Chemistry of Materials, vol. 18, no. 18, pp. 4354-4360, 2006.

[6] P. Dietz and D. Leigh, "Diamondtouch: A multi-user touch technology," in UIST '01, 2001, pp. 219-226.

[7] A. Dix, Human-Computer Interaction. Prentice Hall, ESM Marketing-Computer Science, 2004.

[8] E. D. Gates, Introduction to Electronics. Thomson Learning, 2006.

[9] S. Greenberg and C. Fitchett, "Phidgets: easy development of physical interfaces through physical widgets," in UIST '01, 2001, pp. 209-218.

[10] T. Grosse-Puppendahl, E. Berlin, and M. Borazio, "Enhancing accelerometer-based activity recognition with capacitive proximity sensing," in $A m I$ ' 12,2012 , pp. 17-32.

[11] T. Grosse-Puppendahl, A. Marinc, and A. Braun, "Classification of user postures with capacitive proximity sensors in aal-environments," in AmI '11, 2011, pp. 314-323.

[12] V. Pavlovic, R. Sharma, and T. Huang, "Visual interpretation of hand gestures for human-computer interaction: a review," IEEE Transactions on Pattern Analysis and Machine Intelligence, vol. 19, no. 7, pp. 677-695, Jul. 1997.

[13] M. Sato, I. Poupyrev, and C. Harrison, "Touché: enhancing touch interaction on humans, screens, liquids, and everyday objects,' in CHI '12, 2012, pp. 483-492.

[14] V. Savage, X. Zhang, and B. Hartmann, "Midas: Fabricating custom capacitive touch sensors to prototype interactive objects,' in UIST '12, 2012.

[15] J. R. Smith, "Field mice: extracting hand geometry from electric field measurements," IBM Systems Journal, vol. 35, no. 3-4, pp. 587-608, 1996.

[16] J. R. Smith, "Electric Field Imaging," Ph.D. dissertation, Massachusetts Institute of Technology, 1999.

[17] A. Steinhage and C. Lauterbach, "Sensfloor (r): Ein AAL Sensorsystem für Sicherheit, Homecare und Komfort," in $A A L$ Kongress '08, 2008, (in German).

[18] M. Valtonen, J. Mäentausta, and J. Vanhala, “Tiletrack : Capacitive human tracking using floor tiles,' in PerCom '09, 2009, pp. 121-133.

[19] R. Wimmer, M. Kranz, S. Boring, and A. Schmidt, "A capacitive sensing toolkit for pervasive activity detection and recognition," in PerCom '07, 2007, pp. 171-180.

[20] R. Wimmer, M. Kranz, S. Boring, and A. Schmidt, "Captable and capshelf - unobtrusive activity recognition using networked capacitive sensors,' in INSS '07, 2007, pp. 85-88.

[21] T. G. Zimmerman, J. R. Smith, J. A. Paradiso, D. Allport, and N. Gershenfeld, "Applying electric field sensing to humancomputer interfaces," in CHI '95, 1995, pp. 280-287. 